\title{
[ 205$]$
}

\section{V.}

Beobachtungen über die Klangfiguren auf ebenen nach allen Dimenfionen fchwingenden homogenen Scheiben;

v 0 II

Strenzke, zweitem Lehrer d. Math. a. Gymn. zu Danzig.

Die fichere Hervorbringung der Klangfiguren hängt ab von der Walal der klingenden Körper, von dem beweglichen Mittel, welches an gewiffen Stellen der bewegten Oberfläche zur Ruhe konmt und von der fichern Anfftellung der tönenden Scheiben. Immer wird hier voransgefetzt, dafs die Erregung der Sclivingungen durch einen Violinbogen gelchieht.

Zur Hervorbringnng der Klangfiguren fcheinen fich vorzüglich möglichft genan gearbeitete Scheiben von Meffing oder Glockenmetall zu eignen, von folclier Dicke, dals die Enden der Scheibe nicht in befondere, von den Hauptfchwingungen unablıängige, Schwingungen verfetzt werden können. Die Seitenlänge der von mir gebranchten Quadrat cheiben betrug 4 bis 6 Zoll Rheinl. bei einer Dicke von $\frac{x}{2}$ bis $\frac{3}{4}$ "'”.

Den Gebrauch der Glasfcheiben kann ich nach meinen Erfahrungen weniger empfehlen, wenigftens dann nicht, wenn es darauf ankönmt, möglichft beftimmte Figuren zur fichern Beobachtimg hervorzubringen. Denn nicht zu gedenken der gellenden Tö- 


\section{[ 206 ]}

ne, welche t5nende Glasfcheiben entwickeln, ein Un1ftand, der freilich nicht für jeden Beobachter und jede Umgebung gleich laftig wird, fo geben gläferne Scheiben nie fo reine und fcharf begranzte Figuren, als metallene, zumal folche, die ein lingerer Gebranch fahig gemacht hat, volle und reine Töne anz.ngeben. Dafs jene Scheiben minder ficharfe Figuren hervorbringen, mag vielleicht in der ungleichartigen Zufammenfetzung der kleinften Theile des Glafes feinen Grund haben, nicht, wie es mir frülıer erfchien, in der durch die Schwingungen der Sclieibe erregten Elektricitat, welche die Bildung der Figuren auf einem Nichtleiter hindere, auf einer leitenden Metallfcheibe ohne fiörenden Einflufs bleibe; denn auch ifolirte Metallfcheiben zeigen gleich regelmålsige Bildungen. Uebrigens fcheint die von Vielen bei der Bildung der Klangfiguren bemerkte Elektricităt nicht von der innern Bewegung des folıwingenden Körpers abzuhängen, fondern von der Reibung des mit Harz beftrichenen Violinbogens, was um fo wahrfcheinlicher wird, da fie ftets als negative auftritt.

Zur leichtern Orientirung in den Unterftützungspunkten und in den Stellen, wo die Scheibe durch den Bogen in Schwingung gefetzt werden foll, verfieht man das Metall mit einem feinen Netze fenkrecht einander durchlchneidender Linien, welche felbft bei anfehnlicher Tiefo die regelmäl'sige Bildung der Figuren nicht hindern, wio die Vergleichung mit ganz polirten Sclieiben zeigt.

Die Bildung der Klangfiguren ift überdiefs noch durch die fichere Anffellnng der Scheiben bedingt. Die Scheiben mit den Fingerfpitzen feftzuhalten, ifi 


\section{$[207]$}

zunachft felır unbequem, macht abhängig von der individuellen Struktur der Finger, deren gröfsere oder geringere Oberflache njcht ohne Einflufs auf die Hervorbringung der Figuren feyn kamn; und möchie fchon del'shalb nicht rathlam feyn, weil durch dio Finger dem Beobachter ein Theil der ruhenden Linien verfteckt wird. Aber diele Art der Auffellung wird logar ummöglich in den dem Pando der Scheibe mahe gelegenen Punkten, wo die halienden Finger nicht hinlăngliche Kraft befitzen, um der am entgegengefutzten Ende der Scheibe, allo am längern Hebelarme wirkenden Kraft des Bogens das Gleichgewicht $2 u$ halten.

Eine fehr fichere Auffellung dor Klangfcheiben wird erreicht durch eine Art eiferner Gabel mit hölzernem Griffe, im Velentlichen der obere Theil der von Hrr. Chladni zu diefen Verfuchen vorgefchlagenen Scliraube mit einigen Abünderungen. (Fig. 23. Taf. IV.)

Die beiden Schenkel der Gabel find aus ftarkem Eifen verfertigt. Die Länge eines jeden Schenkels beträgt in meinem Exemplare 4 Zoll, die Breite $\frac{1}{4}$, die Höhe eben fo viel. Der obere Schenkel endigt fich in einen Cylinder, durch welchen eine Schraube geht, die an ihrem obern Ende eine geriffte Kreisplatle von 1: Zoll Durchmefer trügt. Der untere Theil der Schranbe drückt auf eine Feder, welcho anı obern Theile des Verbindungsftücks der beiden Schenkel durch kleine Schrauben feftgehalten wird. Der obere Theil, der in eine kloine Kreisplatte fich endigenden Fedor nimmt in einer Vertiefung dio Schuabe auf, der untere Theil trägt eine kleine 


\section{[ 208 ]}

Tuchfcheibe von etwa $2^{\prime \prime}$ Durchmeffer. Eine gleich grofse Tuchfcheibe befindet fich, diefer gerade gegenüber auf einem kleinen metallenen Cylinder, welcher fich in den obern Theil des untern Schenkels einfchrauben läfost. Man darf nicht vorgelfen, fowohl die Feder, als dielen Cylinder fo einrichten zu lafen, dars fie beide leicht herauggenommen werden können. Denn bei einem längern Gebrauche haften dio zum Beftreuen angowandten Sandkörner fo feft an den Tuchfcheiben, dafs fie bei jeder Fefittellung der Scheibe das Metall an diefer Stelle der Politur berauben. Diefer Umftand würdo noch läfiger feyn ohne den Gebrauch der Feder, anf welche die Schranbe ilhre Wirkung äufsert. Denn die Sandkörnchen, welche jetzt im fchlinmfien Falle nur leichto Eindrüeke in einzelnen Punkten des Metalls zurücklaffen, würden, bei unmittelbarer Befeftigung der Tuclifcheibe an der Schraube, kreisförmige Verletzungen auf der Scheibe hervòrbringen. Der hölzerne Oriff der Gabel ift in der Figur nur angedentet worden,

Die erwähnten Vorfichtsmalsregeln hatten zum Zweck, die wohl unterftützte Klanglcheibe in freie Schwingung zu verletzen; jetzt mögen die beweglichen Mittel betrachtet werden, welche dazn dienen, die durch die entgegengefetzten Schwingungen zur Ruhe gebrachten Stellen dem Auge fichtbar zu machen. Dazu können aber fowohl kleine Theilchen fefter Körper als Flülfigkeiten dienen. Unter den feften Körpern l'cheint der reine ftanbfreie Quarzand und noch mehr der faliwere magnetifche Fil'enfiud, welcher fich an den Küften des Meeres und an den Ufern der Binnonfeen der Offfeeländer vorlindet, den 


\section{[ 209 ]}

Vorzug zu verđuenen. Veniger dürfte der von Hrn. Profefor Oersted *) hierzu vorgefchlagene Pulvis Lycop. tanglich feyn. Denn da diefer zarte Staub fich fo feft an das Metall hängt, dafs er auch bei erfolgter Umkehrung der Scheibe nicht herabfällt, felbft dann nicht, wenn der Schlag eines Hammers anf die Scheibe geführt wird; wie darf man dann erwarten, dafs die Schwingungen der Scheibe an Stellen, wo die ruhenden Linien fich fehr nahe kommen, im Stande feyn werden, den Staub abzuwerfen, der mit fo grofser Kraft an der Scheibe zurückgehalten wird? In der That hat anch die Anwendung jenes Staubes die Bildungen der Figuren verfteckt.

Aber auch der Gebranch des ganz ftaubfreien Sandes auf der forgfältig gereinigten Scheibe erfordert noch einige Vorficht. Denn find $A B$ und $C D$ zwei ruhende Cinien, beide einander fehr nahe: fo wer-
den fie für den zwifchen ihnen liegenden
Sand die Gränzen angeben, über welche ihn
die Bewegung der Scheibe nicht führen
D kann; ift num der Zwifchenraum mit einer dicken Sandfchicht bedeckt, fo wird der Beobachter in der Biegung den Durch fchnitt zweier Linien zu fehen glauben, wo in der That kein Durchlchnitt vorhanden ift. Darum ift es nothwendig, die Scheibe nur mit wenig Sand zu beftreuen, fo viel eben

*) Gehlens Journal für die Chemie, Phyf. u. Mineralogie. VIIf. 223. $O$. bediente fich inders auch des Pulvers anderer Subftanzen, unter welchen er befonders das gepülverte Blei, als dasjenige rühmt, welches die fchärfften Linien giebt. 


\section{[ 210 ]}

hinreicht, die ruhenden Stollen fichtbar zo machen und keine derfelben zu übergehen.

Mit diefen Vorfichtsmaísregeln wird man es nach eincr geringen Uebung in der fichern Führung des Bogens leicht dahin bringen, die Klangfiguren fo rein und zart darzuftellen, dafs kein Sandkörnchen vereinzelt anf der glänzenden Tafel liegt, fondern alle cinzeln an einander ruhen, wie anf cinem nufichtbaren, mathematilchen Faden anfgereihet.

Aulser den feften Körpern können auch Flülfigkeiten, am bequemften Waffer, in einer dünnen Schicht über die Scheibe gegofen, zur Bezeichnung der ruhenden Stellen dienen. Dicfes Mittel, welches $\mathrm{Hr}$. VV he atsto ne bekannt gemacht hak, deflen frühefte Anwendung aber von Hin. Chladni herrührt, wio man aus feiner Akuftik fielit, wird olne $Z$ weifel dazu dienen. tiefer in diefe verwickelten Erfcheinnngen der Akuftik einzudringen, weil die Bewegung der Flüifgkeit fich nuaufhörlich wiederholen lüfst. Bei diefer Bewegung ericheint die garze Oberflüche der Flïffigkeit wie mit einem Nelze iiberzogen, welches anfangs weiter ift bei den tiefern Tönen der Scheibe, mit der zunelmenden Hölie der Töne fich zulammenzieht, und bei den liöchlten Tönen, welche die Scheibe hervorbringen kann, dem feinften Gewebe von der regelmälsigfien Bildung gleicht: Diefes fcheinbare Gewebe wird durch die kleinen Wellen hervorgebracht, die fich in verlchiedenen Richtungen durchichneiden. Dabei folgen diefelben fo regelmäfsig anf einander, dal's man die Summe der einzelnen Wellen, welche fich zwilchen zweien anf der Sclseibe eingegrabenen Linien befin- 


\section{[ 2111}

den, fehr genau zahlen kann. Die Verfuche mit einer folchen Wafferfchichnt zeigen auch zugleich dentlich, dafs die ruhenden Stellen durch entgegengefetzte Bewegungen entftehen und dafs die fchwingende Scheibe nach allen 3 Dimenfionen bewegt werde.

Zu einigen befoadern Verfuchen kann auch angefeuchteter Sand mit Vortheil angewendet werden. So lülst fich auf diefe Weife darthun, dals in den Klangfcheiben die ruhenden Stellen durch die Scheibe lenkrecht anf die Oberfläche hindurch gehen. Auch kann diefes Mittel gebraucht werden, um dieSchwingungen eines cylindrifchen Glasgefülses fichtbar zu machen. Ueberzielıt man die innere und änfsere Seito eines gewöhnlichen Trinkglales mit ftark angefeuchtetem Sande, (welches für die innere Seite felır leicht gefchieht durch Drehung des Glales um leine horizontal gelegte Axe, wobei der angefeuchtete Sand fich an der innern Seite des Gefäl'ses anlegt,) und fetzt das Glas durch einen Violinbogen in Sch wingung, fo vertheilt ficle der Sand in 4 oder 6 Dreiecke, welche ihre Spitzen in dem Rande des Glafes haben, während die übrigen Stellen defelben frei und durchfichtig werden (Fig. 22. T. IV.). Anf der äufsern Seite erfcheint eine gleich grofse Anzahl ruhender Stellen, welche aber zwifchen den ruhenden der innern Scile liegen, niclıt diefe decken, wie es bei ebenen Scheiben der Fall ift.

Wendet man das oben beigebrachte Verfahren an, die ruhenden Stcllen einer fchwingenden Scheibe durch trockenen, fiaubfreien Quarz oder Eifenfand fichtbar zu machen, fo wird man fich von der Wahrheit folgender Beobachtungen übcrzeugen können: 


\section{$\left[\begin{array}{ll}212 & 12\end{array}\right.$}

1. Die Klangfiguren, oder die bei fchwingenden Srheiben in Ruhe bleibenden Stellen der Oberfläche find nicht gerade, fondern fiets krumme Linien, aber Linien im Sinne der Geometrie, keine Flächen.

II. Diefe Linien durchfchneiden fich nicht.

Um diefe Beobachtungen zu erläutern, wähle ich die einfachern Schwingungen einer Quadratfcheibe, bemerke aber zuvor, dafs bei allen folgenden Angaben das Tonverhältnil's abfichtlich übergangen ift; weil eine kleine Aendexung in den Unterftülzungspunkten eine wefentliche Aenderung der Figur hervorbringen kann, olne den Ton zu veründern. Defshalb ift es vorgezogen worden, zwei Seiten des vertikal vor dem Beobachter gedachten (Quadrats als ein Axenfyftem anzulehen, deflen Anfangspunkt an dem linken Ende der untern Seite liegt, und die Lage des Unterftützungspunktes der Sclicibe durch die ziz diefen Axen parallele Coordinaten $A, B$, den Punkt der Schwingungsanrogung durch $a, b$ zu bezeichnen, wo die zur Einheit angenommene Seite des Quadrats, bei Rechtecken die lïngere Seite, als Maafs der Coordinaten gilt. In den Zeichnungen dentet das Zeichen o die Stelle der Schwingungsanregung an *).

Legt man durch den Mittelpunkt der Scheibe zwei den Axen parallele Linien, wie in Fig. 2, Taf.III, und unterftützt in der Näle des Mittelpunktes einen Punkt der Scheibe, der zwilchen den boiden Schen-

") Die mit Ch. bezeichneten Figuren find die aus Hrn. Chladni's Akuftik entlehnten hieher gehörigen. 


\section{[ $\left.\begin{array}{lll}2 & 15 & \end{array}\right]$}

keln eines der 4 rechten Winkel liegt, wobei es gleichgültig ift, welche der 4 Ecken des Quadrats in Schwingung verfetzt wird; fo erfcheint entweder Fig. 1 oder 1 *

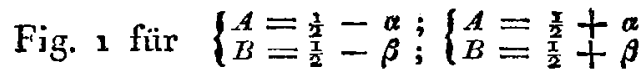

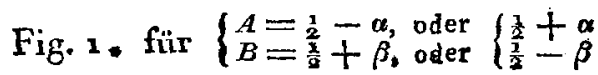

wo $\alpha$ und $\beta$ die Veränderungen der Coordinaten ans. drücken, und ftets pofitiv zu nehmen find.

Wenn man die erfte Figur mit + , die zweite mit - bezeichnet; lo giebt die Anficht der zweiten Figur im Allgemeinen dic Veränderung der Lage an.

Die Gröfsen a und $\beta$ find in gewiflen Gränzen enthalten, zwifchen denen die Kurve, eine Art $\mathrm{Hy}_{\mathbf{s}}$ perbel, fich bewegt. Wird a verkloinert, fo rücken die Schenkel der Kurve fich felbft und dem Mittel. punkte näher bis ż einer gewillen Gränze, von welcher an die Kurven nicht mehr, wie bis dahin durch den Untcrftittzungspunkt gehen. Zungleich tritt die Unmöglichkeit ein, voraus zu beftimnen, ob die Figur die Bildung 1 oder $1 *$ annehmen werde, nnd es zeigt fich in diefem Falle diefelbe Schwingung, welche Hr. Savart bei den Longitudinallchwingungen freiwillige (spontané) Schwingungsart genannt hat. *)

\#) Die Figur I und i, kana auch fo hervorgebracht werden, dafs man 3 Seiten des Quadrats in der Mitte des Randes etwa mit den Fingern unterftützt, und eine der 4 Ecken des Quadrats in Schwingui g verfetzt. Auch hier läfst fich nicht vorausbeftimmen, welche Lage die Kurve annehmen werde; aber Anwa1, d. Pby fik, B.80, Sta 2, J61825. Si。6。 


\section{[ 224 ]}

Bei derfelben Unterftützung und derfelben Stello der Schwingungserregung erl'cheint, wenn abfichtlich die Scheibe in fchnellere Schwingungen verfetzt wird, Fig. 3, von welcher diefelben Bemerkungen über die Lage gelten *).

Wenn die Quadratfcheibe durch 2 Diagonalen in 4 kongruente Dreiecke zerlegt wird, fo erhält man, wenn ein Punkt in einem der $4 \mathrm{um}$ den Mittelpunkt gebildeten rechten Winkel unterftützt wird, entweder Fig. 1 oder 1 * Taf. IV;

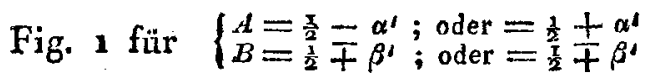

Fig. 1* für $\left\{\begin{array}{l}A=\frac{x}{2} \mp \alpha^{\prime} \text { oder }=\frac{1}{2} \mp \alpha^{\prime} \\ B=\frac{1}{2}+\beta^{\prime} \text { oder }=\frac{2}{2}=\beta^{\prime}\end{array}\right.$

Hier gelten von $\alpha^{\prime}$ und $\beta^{\prime}$ diefelben Beftimmungen, wie bei Frg. 1 und $1_{*}$ Taf. III. Die Lage lälst fich über gewife Gränzen hinaus nicht vorher beftimmen, eben fo wenig dann, wemn 3 Ecken des Quadrats unterftützt werden, während einer der 4 Seitenmittelpunkte die Schwingungen empfüngt. Fig. 3 ift analog der Figur 3 Taf. III; Fig. 2 Taf. IV giebt das Schema der Veränderungen.

Anf die Figuren 1 und ${ }_{*}$, Taf. III und IV laffen fich nun viele Klangfiguren zurückführen, unter der

jeder gegebenen Scheibe kommt entweder Fig. I oder I* zu, und keine Seite der Scheibe verwechrelt jemals die an ihr beobachtete Figur.

") Für diejenigen, welche diefe Verfuche anftellen wollen, bemerke ich noch, dafs die Hervorbringung der Fig. I und I* nicht ohne einige Schwierigkeit in, dafs aber die folgenden Figuren darzußellen, keine Mühe macht. 


\section{$\left[\begin{array}{ll}215 & 1\end{array}\right]$}

Voransfetzung, dafs, wenn in einem Syfteme kongruenter, feî́ mit einander verbundener Scheiben eine Scheibe eine gewifle Schwingung erhält, fich dicie allein Scheiben des Syftems mitheilt, wodurcli eine cinfache Schwingung fich genan 4 oder $9 \mathrm{Mal}$ wiederholen kann, wenni das Quadrat als ans 4 oder 9 kongruenten Quadraten beftehend angefehen werden kamn. Doch ift liebei die Tlieilung in Rechtecke nicht ausgelchlolfen. Die jedes Mral nach der obigen Anficht wahrfelieinlichen Theilungen find in den Zeichnungen durch punktirte Linion angedentet, kömnen aber anch ganz übergangen werden; weil die Figuren Darfellungen der durch die Erfahrung ansgenittelten Klangfigtren find, nicht folche, die fich aus jener Anficht durch Zufammenletzung und Viederliolung der cinfachen Formen ergeben.

Nehmen wir cin Rechteck an, wie Fig. 5 T. III, in welchen die Sciten fich wie 1 : 2 verhalten, fo erfcheint Fig. 5 T. III oder Fig. $5 *$, je nachdem das untere Quadrat die der Fig. 1 oder $1 *$ zukommende Unterftützung erlialt. Fig. 6 liefert das Schema der Veränderungen, und zeigt zugleicl die 6 Stellen der Schwingungsanregung.

Auf der Gränze der Figuren + und - zeigt fich dic freiwillige Schwingungsart, die in Fig. 7 und 7 * dargeftellt ift.

Das aus 2 Quadraten zufammengefetzte Rechteck werde nun durch Hinzufügung zweier Rechtecke zu 


\section{[ $\quad 216$ ]}

oinem Quadrate erganzt, wio diefs in der dritten Quadratreihe auf Taf. IV angedeutet ift, dann erhalt man

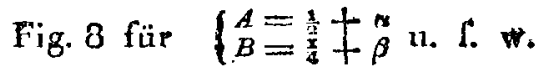

Fig. 8, für $\left\{\begin{array}{l}A=\frac{3}{B} \overrightarrow{+} \dot{\beta} \\ B=\frac{1}{4}\end{array}\right.$ n. $\int$. w.

Fig. 9 oder 9 * auf der Gränze von + und - Tig. 19 bei derfelben Unterfï̈̈tzung und derfelben Stelle der Schwingungserregung, wemn die Schwingungen fchneller werden, und ficherer, wenn noch ein Punkt unterftützt wird, deffen $A^{\prime}=\frac{7}{8}, B^{\prime}=\frac{\pi}{4}$ etc. Anf der Gränze von Fig. 12 und 12 . zeigt fich Fig. 15 und Fig. ${ }^{3} 3_{*}$, die hier nicht verzeichnet ift ${ }^{*}$.

Um Fig. $1 \tilde{\mathfrak{j}}$ zu erhalten, wird man cinen Pnnkt

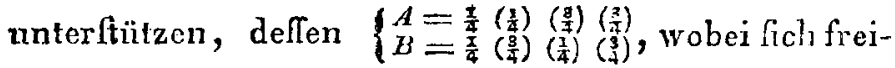
lich nur die Lago der Figur auf dem qten Theile der Scheibe wird voraus befimmen lafien, indem die andern Theile entweder die Schwingung 1 oder $3_{*}$ T. III machen können. Dadurch entfteht eine gröfsere Mannigfaltigkeit von formen, welche liser für den Fall dargefiellt find, wenn

$$
A=\frac{3}{4}+\stackrel{\alpha}{+}+\beta
$$

-) Die Umkehrung der Figuren låst fich befonders leicht fir diefe Stelle der Scheibe wahrnehmen; man hat aber nicht nöthig, um Fig. 8 * darzuftellen, die Unterfützung zu verändern, indem die untere Seite der Scheibe Fig. 8 * giebt, wenn die obere Fig. 8 zeigt. 


\section{$\left[\begin{array}{ll}227 & 1\end{array}\right.$}

docls mufs ich bemerken, dafo Fig. a 6 und a 7 mir nicht auf Quadratcheiben, woll aber in Zufammenfetzungen vorgekommen find, wio auf dem Rechtecke Fig. 28, für $\begin{cases}A=\frac{15}{3}, & a=\frac{3}{3} \\ 16 & b=\frac{1}{2}\end{cases}$

Auf der 4ten Tafel find nait Ausnahme der zollen und 2uften Figur dio Schwingungsarten abgebildet, welche zu den Figuren 1 und 1 * gehören. Ein Quadrat, fo unterftützt, dafs

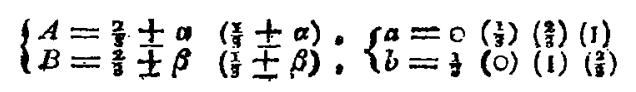

giebt die Yig. 5 , Fig. $5 .$, wenn $a$ fich vergröfsert, wahrend $\beta$ conftant bleibt, wobei die Sclienkel der Kurven fich von einander entfernen, ohne jedoch jemals in gerade Linien, wie Fig. $6 \mathrm{Ch}$. überzugelien. Hier find Fig. 7 und to dio freiwilligen Sch wingungsarten.

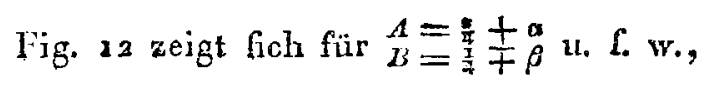

kann aber mit Fig. 12, und andern Bildungen abwecllfeln, welche hier übergangen find; eben lo kann Fig. 23, an die Stelle von 13 treten.

Figur 15 und 26 erfcheint oft unter denfelben Unftänden; die erlte kann aber ftets mit Sicherlieit

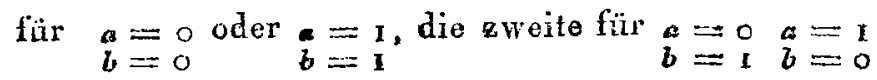
hervorgebrachit werden.

Figur 18 gehört zu einem Punkle, deffer

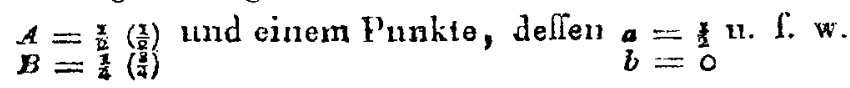




\section{$[218]$}

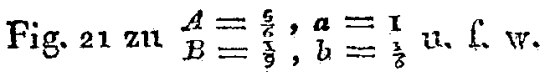

Diefe Figur ift noch deshalb merkwürdig, dafs fich hier die einfachern Solnwingungen der Fig. 1 T. III auf 5 Quadraten mit den zufunmengefetzten Schwingungen yon Fig. 8 T. IV auf den 4 Eckquadraten verbinden.

Diefo Bemerkungen fohcinen linznreichen, um den Sinn jener oben erwahnten Beobachtnngen za erläutern. 